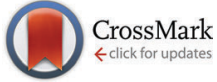

Cite this: Phys. Chem. Chem. Phys., $2016,18,7511$

Received 24th November 2015 Accepted 7th February 2016

DOI: $10.1039 / \mathrm{c5cp} 07238 \mathrm{~g}$

www.rsc.org/pccp

\section{Charging and aggregation of latex particles in aqueous solutions of ionic liquids: towards an extended Hofmeister series}

\author{
Tamas Oncsik, Anthony Desert, $\dagger$ Gregor Trefalt, Michal Borkovec and \\ Istvan Szilagyi*
}

\begin{abstract}
The effect of ionic liquid (IL) constituents and other monovalent salts on the stability of polystyrene latex particles was studied by electrophoresis and light scattering in dilute aqueous suspensions. The surface charge and the aggregation rate were both sensitive to the type of ion leading to different critical coagulation concentration (CCC) values. Systematic variation of the type of IL cation and anion allows us to place these ions within the Hofmeister series. We find that the dicyanoamide anion should be placed between iodide and thiocyanate, while all 1-alkyl-3-methylimidazolium cations can be positioned to the left of the tetramethylammonium and ammonium ions. The hydrophobicity of the 1-butyl-1-methylpyrrolidinium $\left(\mathrm{BMPL}^{+}\right)$ion is intermediate between 1-ethyl-3-methylimidazolium $\left(\mathrm{EMIM}^{+}\right)$and 1-butyl-3-methylimidazolium $\left(\mathrm{BMIM}^{+}\right)$. With increasing alkyl chain length, the 1-alkyl-3methylimidazolium cations adsorb on the latex particles very strongly, and 1-hexyl-3-methylimidazolium $\left(\mathrm{HMIM}^{+}\right)$and 1-octyl-3-methylimidazolium $\left(\mathrm{OMIM}^{+}\right)$lead to pronounced charge reversal and to an intermediate restabilization region.
\end{abstract}

\section{Introduction}

Ionic liquids (ILs) consist entirely of cations and anions, and these systems became the focus of intense research recently, mainly due to their uncommon properties, such as low vapour pressure or wide electrochemical window. ${ }^{1-3}$ These aspects make ILs promising media in material science applications, including energy storage, extraction of minerals or biomolecules and electrodeposition. ${ }^{2-8}$ An important class of materials in these applications are particle suspensions in ILs, as they are relevant in nanoparticle synthesis, catalysis, solar cells, or printing inks. ${ }^{9-13}$ For example, numerous researchers have synthesized novel metal nanoparticles in ILs and they could relate the stability of these suspensions to their catalytic activity. ${ }^{11,14-17}$ In this context, the stability of these suspensions plays a key role, and therefore particle aggregation in ILs was investigated recently. ${ }^{18-23}$

Such particle aggregation studies also focused on IL-water mixtures, and it was quickly realized that on the water-rich side the IL fully dissociates into ions, and that aqueous solutions

Department of Inorganic and Analytical Chemistry, University of Geneva,

30 Quai Ernest-Ansermet, CH-1205 Geneva, Switzerland.

E-mail: istvan.szilagyi@unige.ch; Tel: +4122 3796031

† Present address: École normale supérieure de Lyon, CNRS, Université de Lyon 1, Laboratoire de Chimie (UMR 5182), 46 Allée d'Italie, F-69364 Lyon, France. of ILs closely resemble simple electrolytes. ${ }^{19,20,24}$ A good understanding of the influence of the presence of water in ILs is further important, since most ILs contain water to a certain extent.

Studies of colloidal particle aggregation in simple electrolyte solutions have a long history, including the landmark development of the Derjaguin, Landau, Verwey, and Overbeek (DLVO) theory. ${ }^{25}$ This theory predicts, in agreement with experiment, that the aggregation of charged colloidal particles is slow at low salt concentrations, while at higher concentrations it becomes rapid. The sharp transition between these two regimes occurs at the so-called critical coagulation concentration (CCC). The CCC represents an important characteristic concerning the destabilization power of a given salt, or more precisely of the constituent ions. A major achievement of the DLVO theory was to rationalize the Schulze-Hardy rule, which states that multivalent counterions strongly lower the CCC. ${ }^{25-27}$

However, the CCC can be also influenced by other ionic properties than their valence. A well-studied aspect represents the Hofmeister series, which orders ions according to their hydrophobicity. ${ }^{28}$ This series was originally developed to account for the stabilization power of protein solutions, namely

$$
\begin{gathered}
\mathrm{CO}_{3}^{2-}<\mathrm{SO}_{4}^{2-}<\mathrm{H}_{2} \mathrm{PO}_{4}^{-}<\mathrm{F}^{-}<\mathrm{Cl}^{-}<\mathrm{Br}^{-}< \\
\mathrm{NO}_{3}{ }^{-}<\mathrm{I}^{-}<\mathrm{ClO}_{4}{ }^{-}<\mathrm{SCN}^{-}
\end{gathered}
$$




$$
\begin{gathered}
\mathrm{N}\left(\mathrm{CH}_{3}\right)_{4}{ }^{+}<\mathrm{NH}_{4}^{+}<\mathrm{Cs}^{+}<\mathrm{Rb}^{+}<\mathrm{K}^{+}<\mathrm{Na}^{+}< \\
\mathrm{Li}^{+}<\mathrm{Mg}^{2+}<\mathrm{Ca}^{2+}
\end{gathered}
$$

The series indicates that negatively charged proteins form stable solutions even in the presence of high concentration of ions located on the right hand side, while the ions of the left hand side induce their precipitation already at low concentrations. Typically, particle aggregation follows the same series. ${ }^{29-33}$ In particular, negatively charged particles follow the (above) direct Hofmeister series, whereby the ions on the left induce lower CCCs, while the ones on the right, higher ones. On the other hand, positively charged particles follow the (reversed) indirect Hofmeister series. Thereby, the ions on the left lead to higher CCCs, while the ones on the right, to lower ones. The role of divalent ions is more complicated, since their effect on particle aggregation is greatly influenced by the increased valence as described by the Schulze-Hardy rule. ${ }^{25-27}$ Therefore, divalent ions are not considered here.

The position of an ion in the Hofmeister series can be qualitatively correlated with its hydrophobicity or its degree of solvation. ${ }^{34,35}$ Hydrophilic and well-solvated anions, such as $\mathrm{F}^{-}$or $\mathrm{Cl}^{-}$, appear on the left, while hydrophobic and poorly hydrated anions, such as $\mathrm{I}^{-}$or $\mathrm{SCN}^{-}$, on the right. The cations are arranged in the opposite way. The hydrophilic cations, such as $\mathrm{Li}^{+}$or $\mathrm{Na}^{+}$, appear on the right, while the hydrophobic ones, such as $\mathrm{N}\left(\mathrm{CH}_{3}\right)_{4}{ }^{+}$or $\mathrm{NH}_{4}{ }^{+}$, on the left. Many colloidal particles have a hydrophobic surface (e.g., polystyrene latex) and the hydrophobic ions will adsorb more strongly to these surfaces than the hydrophilic ones. Therefore, CCC will be lower in the presence of hydrophobic counterions than in the presence of hydrophilic ones. Conversely, the CCC will be higher in the presence of hydrophobic coions than in the presence of hydrophilic ones. In the latter situation, however, electrostatic repulsion between the coions and the charged particle may lead to very weak adsorption, and these effects may not be noticeable. In general, the CCCs will decrease with increasing hydrophobicity of cations for negatively charged hydrophobic particles (direct Hofmeister series), while they will also decrease with decreasing hydrophobicity of cations for positively charged particles (indirect Hofmeister series). The reverse argumentation applies to hydrophilic particles (e.g., silica). These trends have been confirmed by CCC measurements in numerous systems experimentally. ${ }^{29,31,32,36-38}$

In the present study, we investigate charging and aggregation of polystyrene latex particles in aqueous solutions of ILs. From the concentration dependence of the aggregation rate, one can extract the CCC, and the observed sequences in these quantities can be used to place the IL constituents into the Hofmeister series. The present investigation is related to an earlier study published by us, which focused on simple monovalent ions only. ${ }^{29}$ That study used the same particles as the present one, thus facilitating a direct comparison of both. While ion specific effects on protein solubilisation and enzymatic activity in aqueous solutions of ILs were studied with a similar aim, ${ }^{39-43}$ we believe that the determination of the CCC for uniform particles provides a reliable measure concerning the position of IL constituents within the Hofmeister series.

\section{Experimental}

\subsection{Materials}

Sulfate and amidine functionalized polystyrene latex particles were purchased from Invitrogen Corporation. The size and polydispersity of the particles were determined by interpreting static light scattering (SLS) data in stable suspensions using Mie theory. ${ }^{44}$ Very good agreement was found with the values obtained in transmission electron microscopy (TEM) measurements by the manufacturer (Table 1). Dynamic light scattering (DLS) yielded slightly higher hydrodynamic radii, probably due to sample polydispersity. The same particles were used in our previous study, ${ }^{29}$ where further properties of these particles are given. Prior to the experiments, the particles were dialyzed against ultrapure water until the conductivity remained constant and below $0.8 \mu \mathrm{S} \mathrm{cm}^{-1}$. For the dialysis, cellulose ester and polyvinylidene fluoride membranes (Spectrum Rancho) were used for the sulfate and amidine modified latex suspensions, respectively. The particle concentrations in the dialyzed stock suspensions were determined by SLS, whereby a calibration curve of the scattering intensity obtained with the original particle suspensions of known concentration was used. Typical concentrations in these stock suspensions were 65 and $7 \mathrm{~g} \mathrm{~L}^{-1}$ for sulfate and amidine particles, respectively. Ultrapure Milli-Q water (Millipore) was used throughout.

ILs used in this study were purchased from IoLiTech and they include 1-butyl-3-methylimidazolium as a cation with chloride (BMIM-Cl), bromide (BMIM-Br), dicyanoamide (BMIM-N(CN) $)_{2}$ and thiocyanate (BMIM-SCN) ions, 1-butyl-1-methylpyrrolidinium ILs as the same anions (BMPL-Cl, BMPL-Br, BMPL-N(CN) $)_{2}$ and BMPL-SCN) and the chloride salts of 3-methylimidazolium (MIM-Cl), 1-ethyl-3-methylimidazolium (EMIM-Cl), 1-hexyl-3methylimidazolium (HMIM-Cl) and 1-octyl-3-methylimidazolium cations (OMIM-Cl) (Fig. 1). The ILs were dried under vacuum at $50{ }^{\circ} \mathrm{C}$ for one day and Karl-Fischer titration (Metrohm) was performed to determine their final water content, which was always below $1 \mathrm{~g} \mathrm{~L}^{-1}$. The dried ILs were handled in a glove box. In some cases, mixing the ILs with water resulted in the formation of precipitates due to the presence of impurities, which could be detected by light scattering. These precipitates were removed by allowing the aqueous solutions to stand overnight and filtering with a $0.1 \mu \mathrm{m}$ syringe filter (Millipore). Inorganic salts of analytical grade were purchased from Sigma Aldrich $\left(\mathrm{NaCl}, \mathrm{NaN}(\mathrm{CN})_{2}\right.$ and $\left.\mathrm{NaSCN}\right)$ and Fluka $(\mathrm{NaBr})$. Their solutions were prepared by mixing the calculated amount of solid salt with ultrapure water. All stock solutions and water

Table 1 Characteristic size values of the sulfate and amidine functionalized polystyrene latex particles used in the present study

\begin{tabular}{lllllll}
\hline & \multicolumn{2}{c}{ Average radius (nm) } & & \multicolumn{2}{c}{ Polydispersity $^{c}(\%)$} \\
\cline { 2 - 3 } Latex particles & TEM $^{a}$ & SLS $^{b}$ & DLS $^{b}$ & & TEM $^{a}$ & SLS $^{b}$ \\
\hline Sulfate & 265 & 263 & 278 & & 2.0 & 3.8 \\
Amidine & 110 & 110 & 117 & & 4.3 & 7.1
\end{tabular}

${ }^{a}$ Measured by the manufacturer. ${ }^{b}$ Determined in stable suspensions.

${ }^{c}$ Coefficient of variation. 


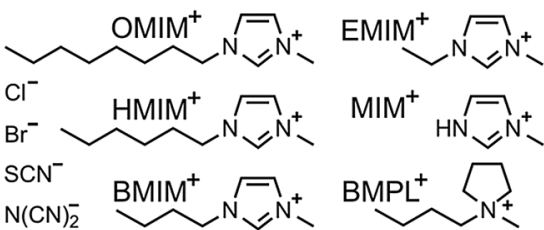

Fig. 1 Chemical structure of IL constituents used in the present study. The anions include chloride, bromide, thiocyanide and dicyanoamide with methylimidazolium, 1-alkyl-3-methylimidazolium and 1-butyl-1-methylpyrrolidinium as cations.

were adjusted to $\mathrm{pH} 4.0$ with $\mathrm{HCl}$ and filtered prior to sample preparation. The measurements were carried out at a temperature of $25.0 \pm 0.2{ }^{\circ} \mathrm{C}$.

\subsection{Electrophoresis}

A ZetaSizer Nano ZS (Malvern) instrument was used to determine the electrophoretic mobility of the particles. During sample preparation, water was mixed with the appropriate volume of stock electrolyte or IL solutions to reach the desired concentration. The particles were then added from the concentrated stock suspension to get final particle concentrations of $5 \mathrm{mg} \mathrm{L}^{-1}$ in the case of amidine and $50 \mathrm{mg} \mathrm{L}^{-1}$ for the sulfate latex. The samples were equilibrated for one minute in the instrument prior to the measurements. Five repetitions were performed and averaged.

\subsection{Particle aggregation}

Time-resolved DLS was used to follow the aggregation process in aqueous particle suspensions. This technique has proved to be most suitable to determine aggregation rates of colloidal particles. ${ }^{45-47}$ The instrument used was an ALV/CGS-3 goniometer (ALV) system, equipped with a He/Ne laser of a wavelength of $633 \mathrm{~nm}$ and an avalanche photodiode as a detector. Samples were prepared in borosilicate glass cuvettes (Kimble Chase). Before the measurements, the cuvettes were cleaned in piranha solution, which is a mixture of concentrated $\mathrm{H}_{2} \mathrm{SO}_{4}$ (Carlo Erba) and $30 \% \mathrm{H}_{2} \mathrm{O}_{2}$ (Reactolab) in a volume ratio of $3: 1$. Subsequently, they were washed with water and dried in a dust-free oven at $60{ }^{\circ} \mathrm{C}$. The particle concentrations were varied in the range of $2-10 \mathrm{mg} \mathrm{L}^{-1}$ for the amidine latex and 50-200 $\mathrm{mg} \mathrm{L}^{-1}$ for the sulfate latex, which corresponds to the number concentration range of $(0.3-2.0) \times 10^{15} \mathrm{~m}^{-3}$. To start the aggregation experiment, the particle stock suspension was injected into a cuvette containing the respective salt solution, and the sample was mixed and inserted in the light scattering system. The correlation function was recorded for 20 seconds at a scattering angle of $90^{\circ}$ and the second-order cumulant fit was used to determine the hydrodynamic radius. The change in this quantity was followed in 50-100 subsequent runs. To probe the early stages of the aggregation, the hydrodynamic radius values never increased more than $40 \%$ in these experiments. This increase is an adequate compromise between good measurement accuracy and minor interferences of higher aggregates. ${ }^{45,48}$ The apparent dynamic aggregation rate coefficient $\Delta$ was determined from the initial rate of increase

$$
\Delta=\left.\frac{1}{R_{\mathrm{h}}(0)} \cdot \frac{\mathrm{d} R_{\mathrm{h}}}{\mathrm{d} t}\right|_{t \rightarrow 0}
$$

where $R_{\mathrm{h}}$ is the hydrodynamic radius and $t$ is the time. The measured apparent rates were then converted to absolute aggregation rate coefficients $k$ by means of the relation

$$
k=\frac{\Delta}{\Delta_{\text {fast }}} \cdot k_{\text {fast }}
$$

where $\Delta_{\text {fast }}$ is the apparent dynamic aggregation rate coefficient in $1.0 \mathrm{M} \mathrm{KCl}$ solution, where the aggregation is in the fast regime. The absolute aggregation rate coefficient $k_{\text {fast }}$ was previously determined using time-resolved simultaneous static and dynamic light scattering in $1.0 \mathrm{M} \mathrm{KCl}$ solutions. In these measurements, the apparent static rate coefficients were obtained from the initial change of the scattered intensity at several scattering angles and plotted against the apparent dynamic aggregation rates. A linear fit was performed on the data and the absolute aggregation rate coefficient was calculated from the intercept. ${ }^{45}$ The resulting values were $k_{\text {fast }}=(3.3 \pm 0.2) \times 10^{-18} \mathrm{~m}^{3} \mathrm{~s}^{-1}$ for the sulfate latex and $(3.0 \pm 0.2) \times 10^{-18} \mathrm{~m}^{3} \mathrm{~s}^{-1}$ for the amidine latex. $^{29}$ The CCCs were determined from plots of the rate coefficient $k$ versus the salt concentration, whereby straight lines were fitted to the experimental points in the slow and fast aggregation regimes. The uncertainty of the CCC determined by this method is about $10 \%$.

\subsection{Viscosity}

A DV-II Pro viscometer (Brookfield) was used to measure the dynamic viscosities of the IL solutions. The concentration ranges of the monovalent electrolytes were chosen according to the concentrations investigated in the mobility and aggregation measurements, usually up to $1.0 \mathrm{M}$. In this range, the viscosities varied linearly with the concentration, and the values for the individual samples were obtained from a linear fit of the data.

\section{Results and discussion}

Surface charge and aggregation rates of sulfate and amidine modified polystyrene latex particles were investigated in the presence of ILs and monovalent electrolytes by electrophoresis and DLS. The principal aim of these experiments was to place some common IL constituents (Fig. 1) into the Hofmeister series. In addition, the influence of the alkyl chain length within the 1-alkyl-3-methylimidazolium series was investigated.

\subsection{General trends}

Electrophoretic mobilities and aggregation rates of sulfate and amidine latex particles were measured in different ionic environments. Initially, we investigated the effect of anions, namely $\mathrm{Cl}^{-}, \mathrm{Br}^{-}, \mathrm{N}(\mathrm{CN})_{2}{ }^{-}$and $\mathrm{SCN}^{-}$, in the presence of $\mathrm{Na}^{+}$as the cation (Fig. 2). Subsequently, the same anions were investigated in the presence of $\mathrm{BMIM}^{+}$(Fig. 3) and $\mathrm{BMPL}^{+}$(Fig. 4) as cations. In all systems, the electrophoretic mobility increases with the salt concentration for the sulfate particles and decreases for the amidine particles, sometimes resulting in an isoelectric point (IEP) and a subsequent weak charge reversal. These trends are mainly caused by the progressive screening through the 
(a)

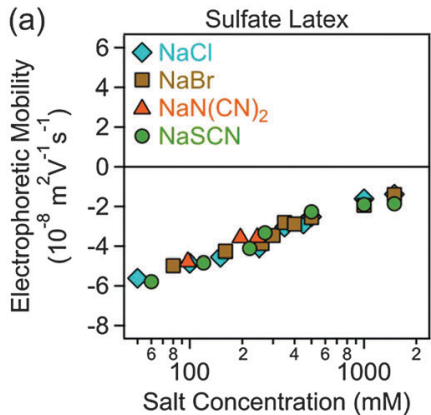

(b)
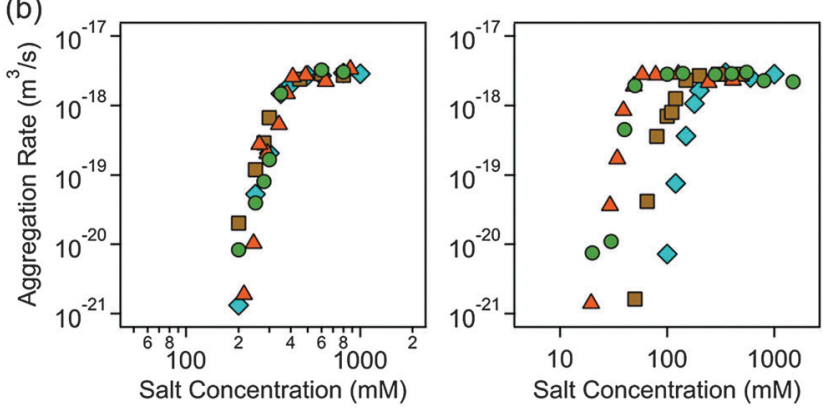

Fig. 2 Electrophoretic mobility (a) and absolute aggregation rate (b) for sulfate (left column) and amidine (right column) latex particles as a function of the salt concentration for different monovalent electrolytes of the sodium cation. The results with the $\mathrm{Cl}^{-}, \mathrm{Br}^{-}$and $\mathrm{SCN}^{-}$ions have been already published earlier. ${ }^{29}$
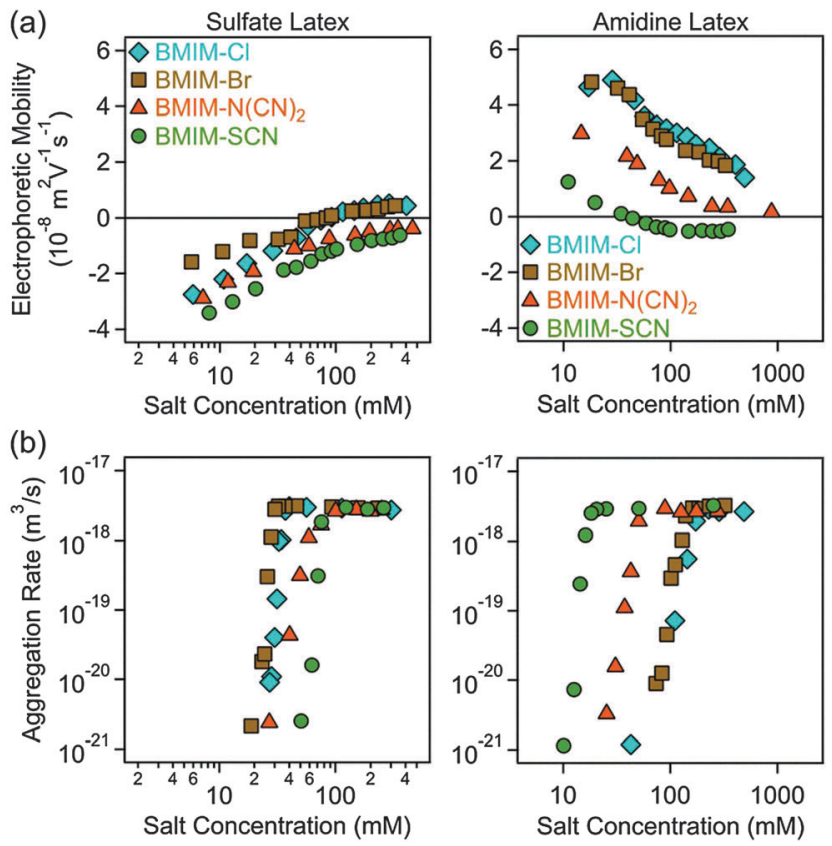

Fig. 3 Electrophoretic mobility (a) and absolute aggregation rate (b) values of sulfate (left column) and amidine (right column) latex particles as a function of the salt concentration in aqueous solutions of different ILs of the $\mathrm{BMIM}^{+}$cation.

electrolyte and simultaneous adsorption of the counterions. The particle aggregation rates increase rapidly with increasing salt concentration in the slow aggregation regime, and reach a
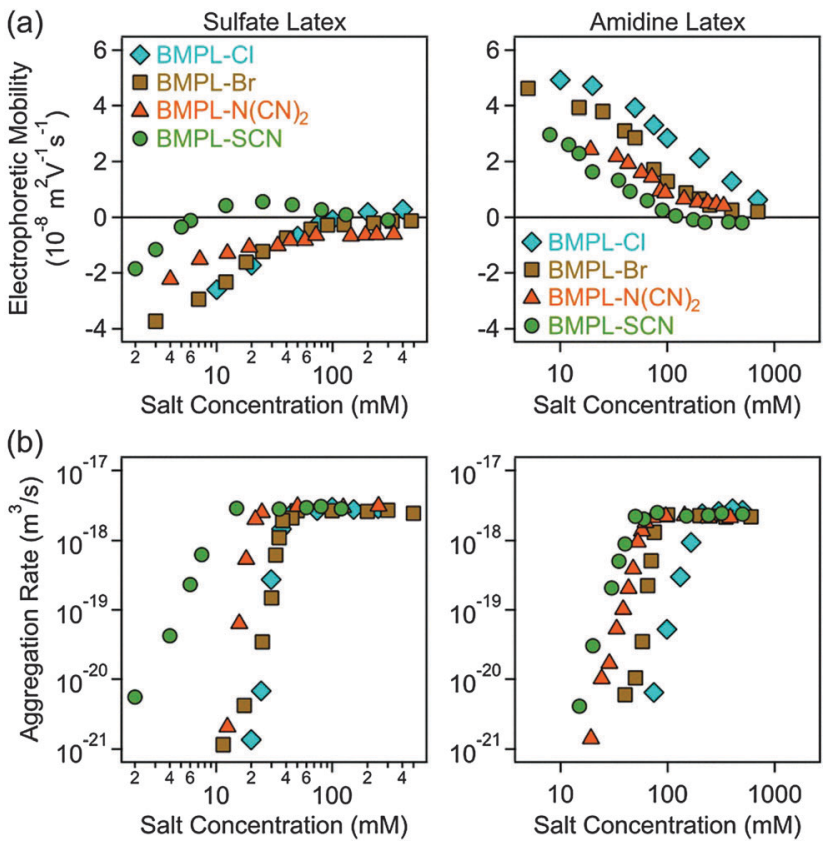

Fig. 4 Electrophoretic mobility (a) and absolute aggregation rate (b) values of sulfate (left column) and amidine (right column) latex particles as a function of the salt concentration for different ILs of the $\mathrm{BMPL}^{+}$cation.

constant value at higher concentrations in the fast aggregation regime. The CCC is located in the narrow transition zone between these two regimes. This behaviour is typical for charged colloidal particles dispersed in electrolyte solutions and can be predicted by the DLVO theory, ${ }^{25}$ in some situations even quantitatively. ${ }^{49,50}$ The characteristic influence of the type of ion present on the CCCs will be detailed below. No clear trends were observed in the dependencies of the aggregation rates on the salt concentration in the slow aggregation regimes.

\subsection{Fast aggregation regime}

The possible influence of the ion type on the fast aggregation rate coefficients measured above the CCCs was addressed. The ions investigated include $\mathrm{Cl}^{-}, \mathrm{Br}^{-}, \mathrm{N}(\mathrm{CN})_{2}{ }^{-}$and $\mathrm{SCN}^{-}$anions, and $\mathrm{Na}^{+}, \mathrm{BMIM}^{+}$and $\mathrm{BMPL}^{+}$cations. As one must consider viscosity effects, the absolute rate coefficients in the fast aggregation regime were normalized by Smoluchowski's rate coefficient for diffusion controlled aggregation ${ }^{51}$

$$
k_{\mathrm{S}}=\frac{8 k_{\mathrm{B}} T}{3 \eta}
$$

where $k_{\mathrm{B}}$ is the Boltzmann constant, $T$ is the temperature, and $\eta$ is the dynamic viscosity of the electrolyte solution. The latter value was measured for the respective salt or IL solutions within the appropriate concentration range. No dependence of the normalized fast aggregation rate coefficients on the type of ion is observed, and one finds very similar normalized coefficients for both types of particles (Fig. 5a). Their values were $k_{\text {fast }} / k_{\mathrm{s}}=0.24 \pm 0.01$ and $0.24 \pm 0.02$ for the sulfate and amidine particles, respectively. These values are comparable to the ones reported earlier for the same particles in the presence of 

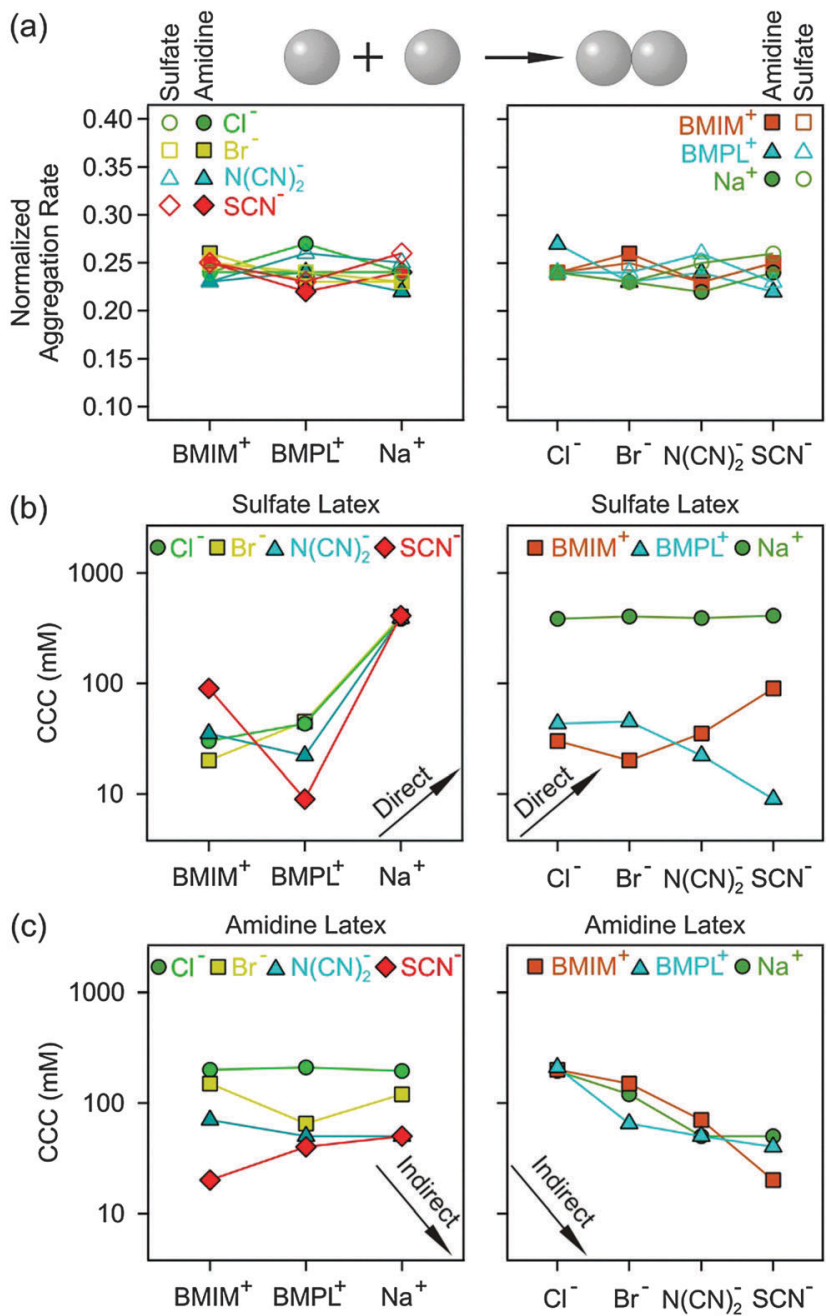

Fig. 5 Normalized fast aggregation rate coefficients (a) and CCC values for sulfate (b) and amidine (c) latex particles in the presence of different cations (left) and anions (right). The arrows indicate the expected trends according to the direct or indirect Hofmeister series. The lines are a guide to the eye.

several simple monovalent electrolytes, which were $0.26 \pm 0.01$ and $0.23 \pm 0.01$ for the sulfate and amidine latex, respectively. ${ }^{29}$ These findings suggest that the attractive forces (i.e., van der Waals and hydrophobic forces), which determine the fast aggregation rates, do not strongly depend on the type of ion present.

\subsection{Ion specific effects}

For negatively charged sulfate latex particles, there were no specific effects of the coions, provided $\mathrm{Na}^{+}$was used as the counterion. All the anions used, namely $\mathrm{Cl}^{-}, \mathrm{Br}^{-}, \mathrm{N}(\mathrm{CN})_{2}{ }^{-}$and $\mathrm{SCN}^{-}$, adsorb to the particle surface only weakly, and therefore the electrophoretic mobility (Fig. 2a), aggregation rates (Fig. 2b) and CCCs (Fig. 5b) remain the same within the experimental error. With the exception of the $\mathrm{N}(\mathrm{CN})_{2}{ }^{-}$anion, the same observation was already reported earlier. ${ }^{29}$ For the amidine latexes, the electrophoretic mobilities decrease with the salt level and their values at the same concentration decrease within the series $\mathrm{Cl}^{-}$, $\mathrm{Br}^{-}, \mathrm{N}(\mathrm{CN})_{2}{ }^{-}$, and $\mathrm{SCN}^{-}$(Fig. 2a). The adsorption of $\mathrm{N}(\mathrm{CN})_{2}{ }^{-}$ and $\mathrm{SCN}^{-}$ions results in an IEP and a charge reversal, suggesting that these ions are rather hydrophobic and that they adsorb on these particles strongly. Similar charge reversal has been observed with other less solvated monovalent ions. ${ }^{29,34,52}$ The trend in the mobilities is also reflected in the CCCs (Fig. 2b and $5 \mathrm{c}$ ). One observes that the CCC decreases in the same sequence as stated above, namely for $\mathrm{Cl}^{-}$being the highest and for $\mathrm{N}(\mathrm{CN})_{2}{ }^{-}$and $\mathrm{SCN}^{-}$the lowest. This order can be explained as follows. The hydrophobic counterions, such as $\mathrm{N}(\mathrm{CN})_{2}{ }^{-}$and $\mathrm{SCN}^{-}$, adsorb strongly on the hydrophobic particle surface, leading to a decrease of the surface charge and to lower CCCs. On the other hand, the hydrophilic $\mathrm{Cl}^{-}$counterion adsorbs weakly leading to highly charged particles, which also have higher CCCs. The observed sequence $\mathrm{Cl}^{-}>\mathrm{Br}^{-}>\mathrm{SCN}^{-}$ reflects the expected indirect Hofmeister series, as reported before. ${ }^{29}$ The new finding here is that $\mathrm{N}(\mathrm{CN})_{2}{ }^{-}$behaves similar to $\mathrm{SCN}^{-}$. This observation agrees with earlier protein precipitation experiments. ${ }^{42}$

When the $\mathrm{BMIM}^{+}$counterion was used, electrophoretic mobilities and aggregation rates of the negatively charged sulfate latex particles were strongly influenced by the type of coion (Fig. 3a and b). The magnitude of the mobility and of the CCCs (Fig. 5b) was significantly lower in the presence of BMIM ${ }^{+}$ than for $\mathrm{Na}^{+}$, confirming the considerable adsorption of the $\mathrm{BMIM}^{+}$counterions to the oppositely charged surface. In these systems, the mobilities and CCCs were sensitive to the type of coion, whereby the CCCs increase in the sequence $\mathrm{Br}^{-}<$ $\mathrm{N}(\mathrm{CN})_{2}{ }^{-}<\mathrm{SCN}^{-}$. With the exception of the $\mathrm{Cl}^{-}$ion, this trend reflects the expected direct Hofmeister series for negatively charged hydrophobic surfaces. ${ }^{33}$ This finding is in line with recent colloidal probe experiments with hydrophilic silica particles in the presence of the $\mathrm{BMIM}^{+}$counterions, which reports the strength of the short-range attraction to increase in the sequence $\mathrm{Cl}^{-}<\mathrm{N}(\mathrm{CN})_{2}^{-}<\mathrm{SCN}^{-}{ }^{-24}$ Such attraction was significant at salt levels close to the CCCs, and therefore the CCC is expected to decrease in the same sequence as stated above in agreement with the indirect Hofmeister series for negatively charged hydrophilic particles.

A similar sequence of counterions was observed for the amidine particles as in the presence of $\mathrm{Na}^{+}$. The mobilities (Fig. 3a) and the CCCs (Fig. 5c) decreased again in the sequence $\mathrm{Cl}^{-}>\mathrm{Br}^{-}>$ $\mathrm{N}(\mathrm{CN})_{2}^{-}>\mathrm{SCN}^{-}$in agreement with the indirect Hofmeister series for positively charged hydrophobic surfaces. ${ }^{33}$ The electrophoretic mobilities remain positive for weakly adsorbing $\mathrm{Cl}^{-}$and $\mathrm{Br}^{-}$ counterions, while the strongly adsorbing $\mathrm{SCN}^{-}$ions induce a charge reversal. However, the IEP is situated at much lower concentration than for the $\mathrm{Na}^{+}$ions, which suggests that the hydrophobic $\mathrm{BMIM}^{+}$coion adsorbs to the particle surface, which in turn induces a stronger co-adsorption of $\mathrm{SCN}^{-}$ions due to ion pair formation on the surface. The formation of ion pairs between cations and anions has been reported in various ILs. ${ }^{53-57}$ The IL constituent ions adsorbed on the particle surface may also form ion pairs with the oppositely charged ions. The formation of such surface ion pairs will then influence the surface charge and also the CCC. For the $\mathrm{N}(\mathrm{CN})_{2}{ }^{-}$ anion, charge neutralization occurs as well, but the adsorption is not strong enough to reverse the particle charge. 
For the $\mathrm{BMPL}^{+}$counterion, specific effects of coions were equally observed for sulfate latex particles. Similar electrophoretic mobilities were measured for $\mathrm{Cl}^{-}$and $\mathrm{Br}^{-}$, however, the presence of $\mathrm{N}(\mathrm{CN})_{2}{ }^{-}$and $\mathrm{SCN}^{-}$ions led to higher mobilities (Fig. 4a) and lower CCCs (Fig. 4b and 5b). This trend is opposite to the one observed for $\mathrm{BMIM}^{+}$, and does not follow the anticipated direct Hofmeister series. This reversal of the Hofmeister series could be related to the counterion affinity to the oppositely charged particles and the extent of ion pairing on the surface. The observed trends in the CCCs indicate stronger ion pairing in the BMIM-SCN system than for BMPL-SCN. For the amidine particles, a weak charge reversal is again observed for the $\mathrm{SCN}^{-}$ ions, but the electrophoretic mobilities remain positive for the other counterions (Fig. 4a). The charge reversal occurred at higher concentration than in the $\mathrm{BMIM}^{+}$system, which may indicate either weaker $\mathrm{BMPL}^{+}$adsorption on the particle surface or weaker ion pair interactions between $\mathrm{BMPL}^{+}$and $\mathrm{SCN}^{-}$ions. Nevertheless, the trends in both the charging and aggregation properties of the amidine particles in the presence of $\mathrm{BMPL}^{+}$and $\mathrm{BMIM}^{+}$counterions are similar. Accordingly, the mobilities at the same concentration as well as the CCCs follow $\mathrm{Cl}^{-}>\mathrm{Br}^{-}>$ $\mathrm{N}(\mathrm{CN})_{2}{ }^{-}>\mathrm{SCN}^{-}$(Fig. 4a and $5 \mathrm{c}$ ). This order is in agreement with the indirect Hofmeister series expected for positively charged hydrophobic particles. ${ }^{31,33}$

The observed trends for the cation dependence are summarized in Fig. 5b and c. For the sulfate latex, the presence of the $\mathrm{BMIM}^{+}$counterion leads to lower CCCs than $\mathrm{BMPL}^{+}$in the presence of simple anions, while the trend is reversed in the presence of hydrophobic $\mathrm{N}(\mathrm{CN})_{2}{ }^{-}$and $\mathrm{SCN}^{-}$anions. This reversal is probably related to the variable extent of ion pair formation in these systems. No trend with CCCs was observed for amidine latex particles when the coions were varied.

Let us now compare the present results with the ones of an earlier study, which investigated the CCCs of exactly the same particles. ${ }^{29}$ In particular, various anions in the presence of $\mathrm{Na}^{+}$ and various cations in the presence of $\mathrm{Cl}^{-}$were investigated. Combining the present results with the ones from that study ${ }^{29}$ enables us to place the IL constituents into the established Hofmeister series (Fig. 6). For sulfate latex particles, the extended Hofmeister series becomes

$\mathrm{BMIM}^{+}<\mathrm{BMPL}^{+}<\mathrm{N}\left(\mathrm{CH}_{3}\right)_{4}{ }^{+}<\mathrm{NH}_{4}{ }^{+}<\mathrm{Cs}^{+}<\mathrm{K}^{+}<\mathrm{Na}^{+}<\mathrm{Li}^{+}$

where the hydrophilic $\mathrm{Na}^{+}$ions typically lead to the highest CCC, while the hydrophobic IL constituents to lower CCC. $\mathrm{BMIM}^{+}$and $\mathrm{BMPL}^{+}$have to be positioned on the left hand side of the series, indicating that they are even more hydrophobic than the $\mathrm{N}\left(\mathrm{CH}_{3}\right)_{4}{ }^{+}$ counterion. The sulfate particles show no effects of coions. The CCCs of amidine latex particles in the presence of different counterions decrease according to the indirect Hofmeister series as

$$
\mathrm{H}_{2} \mathrm{PO}_{4}^{-}>\mathrm{F}^{-}>\mathrm{Cl}^{-}>\mathrm{Br}^{-}>\mathrm{NO}_{3}^{-}>\mathrm{N}(\mathrm{CN})_{2}{ }^{-}>\mathrm{SCN}^{-}
$$

The $\mathrm{N}(\mathrm{CN})_{2}{ }^{-}$counterion has to be placed between $\mathrm{NO}_{3}{ }^{-}$and $\mathrm{SCN}^{-}$. The $\mathrm{BMIM}^{+}$and $\mathrm{BMPL}^{+}$coions have again no influence on the CCC of the amidine particles. However, the $\mathrm{N}\left(\mathrm{CH}_{3}\right)_{4}{ }^{+}$ and $\mathrm{NH}_{4}{ }^{+}$coions lead to systematically lower CCCs, probably, due to specific interactions with the amidine groups.
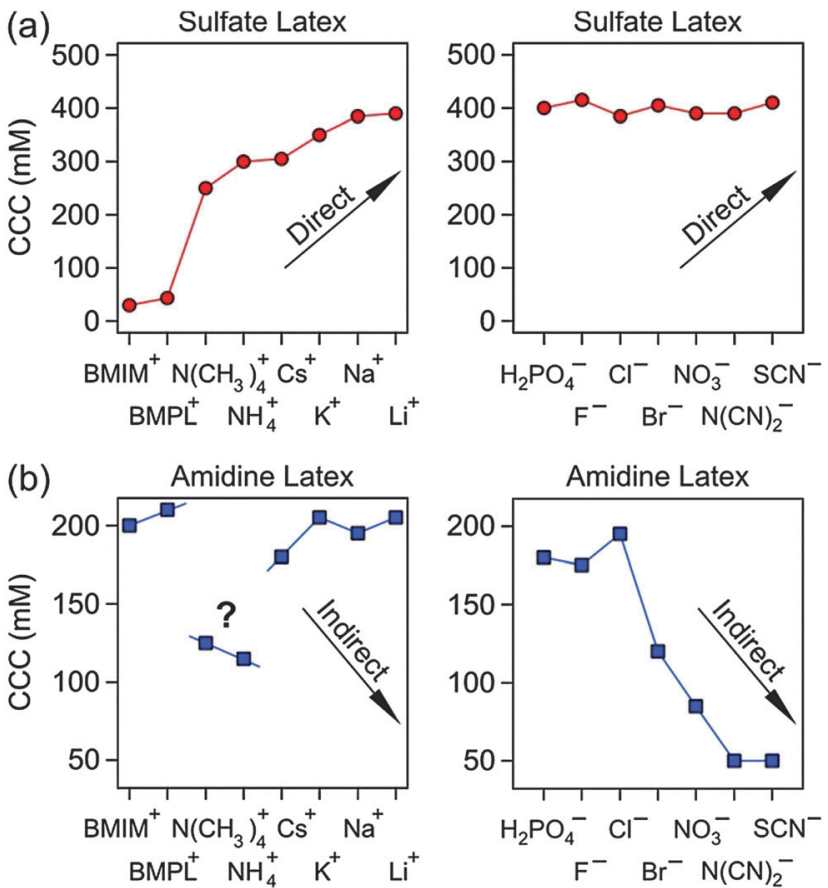

Fig. 6 CCC values for sulfate (a) and amidine (b) latex particles in the presence of different cations (left) and anions (right). The arrows indicate the expected trends according to the direct or indirect Hofmeister series. The lines are a guide to the eye.

\subsection{Effect of the alkyl chain length}

As shown in the previous section, the hydrophobic $\mathrm{BMIM}^{+}$ counterions strongly adsorb on the negatively charged sulfate latex particles and thus modify the particle charge and their CCCs (Fig. 3a and 5b). To further investigate the effects of cation hydrophobicity on charging and aggregation of these particles, we have studied electrophoretic mobilities and aggregation rates of sulfate latex particles in the presence of 1-alkyl-3-methylimidazolium counterions, namely for $\mathrm{MIM}^{+}, \mathrm{EMIM}^{+}, \mathrm{BMIM}^{+}$, $\mathrm{HMIM}^{+}$and $\mathrm{OMIM}^{+}$. Their hydrophobicity increases from the left to the right due to the increasing length of the alkyl chains (Fig. 1). In all cases, the $\mathrm{Cl}^{-}$coion was used.

At sufficiently low concentrations, the electrophoretic mobilities increase with the concentration (Fig. 7a). In the presence of $\mathrm{MIM}^{+}$and $\mathrm{EMIM}^{+}$, the particles remained negatively charged within the entire range investigated. This increase is primarily due to screening by the increasing salt level, but the adsorption of these ions to the particle surface also contributes to this trend. However, adsorption of these counterions becomes more pronounced for longer aliphatic chains and leads to slight charge reversal for $\mathrm{BMIM}^{+}$. The adsorption of $\mathrm{HMIM}^{+}$and $\mathrm{OMIM}^{+}$ counterions becomes even more important and induces charge neutralization and a significant charge reversal. At higher concentrations, the mobilities decrease due to screening, as particularly evident in the presence of the $\mathrm{OMIM}^{+}$cation. A similar charge reversal was already reported for negatively charged kaolinite particles in OMIM-Cl solutions. ${ }^{58}$

The charging behaviour is well-reflected in the respective aggregation rates. For the $\mathrm{BMIM}^{+}, \mathrm{EMIM}^{+}$and $\mathrm{MIM}^{+}$cations 

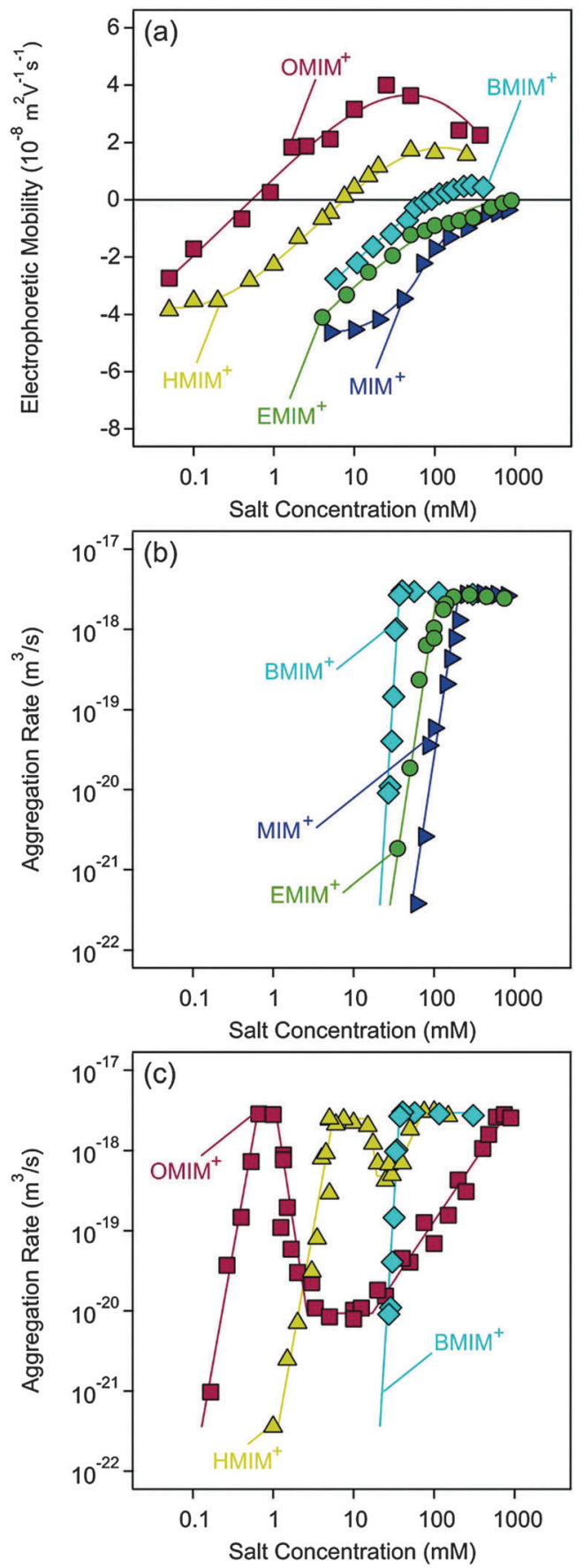

Fig. 7 Electrophoretic mobilities (a) and aggregation rate coefficients (b and $c$ ) of sulfate latex particles in the presence of ILs composed of the chloride anion and the 1-alkyl-3-methylimidazolium cation of different alkyl chains. The lines are a guide to the eye.

with short alkyl chains, the aggregation rates (Fig. 7b) show the classical behaviour of slow aggregation at low concentrations and rapid aggregation at high concentrations, with a CCC in between these two regimes. This situation is similar to the systems discussed above. The CCCs decrease systematically with increasing alkyl chain length, which is caused by the increasing adsorption strength due to increasing length of the alkyl chain.
However, the dependence of the aggregation rate on the IL concentration is notably different in the presence of the $\mathrm{OMIM}^{+}$ counterion (Fig. $7 \mathrm{c}$ ). The aggregation rates are small at low IL concentrations, and they go through a maximum near the IEP. The rate constant at the maximum corresponds to its value in the fast aggregation regime. Upon increasing the IL concentration further, one observes a decrease of the aggregation rate. The rate passes through a minimum and increases again to reach the value in the fast aggregation regime. A similar dependence is observed for the $\mathrm{HMIM}^{+}$counterion, albeit the intermediate minimum is much less pronounced. This shallower minimum is due to the weaker charge reversal of $\mathrm{HMIM}^{+}$as revealed by the electrophoresis. Clearly, $\mathrm{OMIM}^{+}$adsorbs most strongly, and the adsorption strength decreases with decreasing chain length.

One might suspect that the formation of micelles could be relevant in these systems, especially for the cations with a longer apolar alkyl chain. The CMC of OMIM-Cl was reported to be $220 \mathrm{mM}$ and for HMIM-Cl $900 \mathrm{mM} .^{59,60}$ The latter value is at the end of the concentration range used in the present study. Therefore, the formation of micelles does not play any role in the adsorption and particle aggregation mechanism in the HMIM-Cl system. For the OMIM-Cl system, the CMC falls into the upper range of the destabilization due to charge screening by the chloride counterions. Again, micellization plays a minor role.

The observed dependence of the aggregation rate on the concentration of 1-alkyl-3-methylimidazolium can be interpreted in terms of a succession of three CCCs. The first CCC occurs at low concentration during the transition from the slow to fast regime. The second CCC is located after the maximum in the IL concentration, whereby the system undergoes a transition from the fast to slow regime. The third CCC is situated at the highest concentrations after the minimum in the aggregation rate, when the aggregation becomes fast again. Comparing the location of the CCCs with the electrophoretic mobilities in the same concentration ranges, one can realize that the first CCC is caused by charge neutralization, the second is connected with the charge reversal process, while the third CCC is due to the screening effect of the counterions on the surface charge.

A stability map of the CCCs versus the type of counterion of different chain lengths summarizes this characteristic behaviour well (Fig. 8). Three CCCs are observed for $\mathrm{HMIM}^{+}$and $\mathrm{OMIM}^{+}$counterions, while only one CCC is found for $\mathrm{MIM}^{+}$, $\mathrm{EMIM}^{+}$and $\mathrm{BMIM}^{+}$. Therefore, this map shows two regions where the dispersions are stable, meaning that the particle aggregation is significantly slower than that in the case of fast aggregation. That region is indicated as unstable. The first stability region in the lower left corner corresponds to the regular stabilization due to the negative charge of the latex particles. The second region on the right hand side corresponds to the positively charged particles after charge reversal, which is induced by the strong adsorption of the less solvated IL cations. The unstable region in the upper part of the map is due to destabilization and screening at high salt concentrations. The narrow unstable channel in the lower right part of the map is due to the destabilization and charge neutralization at the IEP. The first CCC values at low IL concentration in the presence of 1-alkyl-3-methylimidazolium counterions 


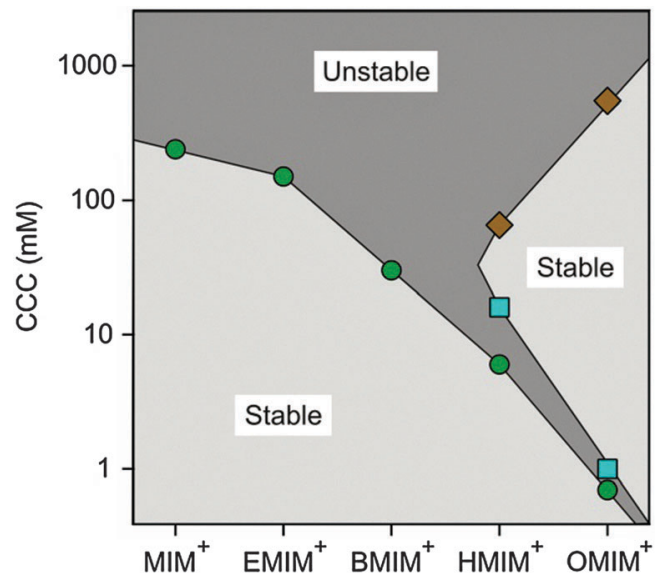

Fig. 8 Stability map including $C C C$ values for sulfate latex particles in the presence of the 1-alkyl-3-methylimidazolium cation based ILs of different alkyl chain lengths with the chloride anion. The circles indicate the first CCC, squares show the second CCC and diamonds refer to the third CCC.

reflect the decreasing chain length and increasing hydrophilicity within the series

$$
\mathrm{OMIM}^{+}<\mathrm{HMIM}^{+}<\mathrm{BMIM}^{+}<\mathrm{EMIM}^{+}<\mathrm{MIM}^{+}
$$

On the left hand side of the series, one finds the hydrophobic cations with a longer alkyl chain. They adsorb more strongly to the particle surface, and induce lower CCCs. Those on the right hand side are more hydrophilic and feature a shorter alkyl chain. The latter counterions adsorb more weakly, and lead to higher CCCs. This trend is reminiscent to previous findings for oxide and silver halide particles in the presence of ionic surfactants of variable chain lengths, whereby the IEP or the first CCC shifts towards smaller concentrations with increasing alkyl chain length. ${ }^{61-64}$ In addition, the restabilization occurred in all systems containing surfactants with an octyl chain or longer. A subsequent destabilization at high concentrations was also observed, quite in analogy to $\mathrm{OMIM}^{+}{ }^{64}$ Enzymatic activity reflects the same order, namely, the proteins lose their activity in the presence of $\mathrm{HMIM}^{+}$at lower concentration than for $\mathrm{EMIM}^{+}$. $^{39,40}$

Similar stability behaviour was reported for the aggregation of latex particles in the presence of multivalent ions or short-chain oligoamines. ${ }^{65,66}$ In particular, the similarity to the system with the oligoamines is striking, and deservers further discussion. ${ }^{66}$ These authors have studied electrophoresis and particle aggregation rates for negatively charged sulfate latex particles in the presence of aliphatic oligoamines with the structural formula $\mathrm{H}_{2} \mathrm{NCH}_{2} \mathrm{CH}_{2}\left(\mathrm{NHCH}_{2} \mathrm{CH}_{2}\right)_{n} \mathrm{NH}_{2}$ for $n=0,1,2$, and 4 . Under the mildly acidic conditions used, these oligoamines form multivalent cations. The concentration dependence of electrophoretic mobilities and of the aggregation rate was very similar to the one shown in Fig. 7, and shows an analogous trend with the increasing chain length. Moreover, the stability map of the CCCs versus the chain length looks surprisingly similar to Fig. 8. Due to these analogies, one might suspect in these two systems that the underlying mechanisms leading to charge reversal and destabilization are similar. The hydrophobic interaction will become more important with increasing chain length and this aspect might be responsible in both systems for the increased extent of adsorption, which would then induce similar patterns in the charging behaviour and aggregation rates. Moreover, these oligoamines are only partially ionized and their charge could be further reduced by complexation of counterions and ion condensation effects. The interesting consequence of this hypothesis is that the multivalent nature of the longer-chain oligoamines becomes secondary and their increasing hydrophobicity determines the adsorption of these molecules and as a consequence, the aggregation of these particles.

\section{Conclusions}

The effect of simple monovalent ions and water-miscible ILs on the surface charge and aggregation of polystyrene latex particles was investigated by electrophoresis and time-resolved light scattering in dilute aqueous solutions. From studies performed on negatively charged sulfate latex particles, the Hofmeister series for the cations may be extended as

$$
\begin{gathered}
\mathrm{OMIM}^{+}<\mathrm{HMIM}^{+}<\mathrm{BMIM}^{+}<\mathrm{BMPL}^{+}<\mathrm{EMIM}^{+}<\mathrm{MIM}^{+}< \\
\mathrm{N}\left(\mathrm{CH}_{3}\right)_{4}^{+}<\mathrm{NH}_{4}^{+}<\mathrm{Cs}^{+}<\mathrm{K}^{+}<\mathrm{Na}^{+}<\mathrm{Li}^{+}
\end{gathered}
$$

The hydrophobic ions shown on the left hand side lead to the lowest CCCs. The most hydrophobic ones, such as $\mathrm{OMIM}^{+}$and $\mathrm{HMIM}^{+}$, show more pronounced charge reversal and a subsequent restabilization. The left hand side of the series reflects the known trend of decreasing hydrophilicity with increasing chain length in surfactant solutions. For amidine latex particles, we conclude that the Hofmeister series for the anions should be extended as

$$
\mathrm{Cl}^{-}>\mathrm{Br}^{-}>\mathrm{N}(\mathrm{CN})_{2}^{-}>\mathrm{SCN}^{-}
$$

whereby the hydrophobic ions are shown on the right hand side and they lead to the lowest CCCs.

\section{Acknowledgements}

The present research was supported by the Swiss National Science Foundation, the University of Geneva and the COST Action CM1206.

\section{References}

1 J. P. Hallett and T. Welton, Chem. Rev., 2011, 111, 3508-3576.

2 A. P. Abbott and K. J. McKenzie, Phys. Chem. Chem. Phys., 2006, 8, 4265-4279.

3 M. Armand, F. Endres, D. R. MacFarlane, H. Ohno and B. Scrosati, Nat. Mater., 2009, 8, 621-629.

4 A. Izgorodin, R. Hocking, O. Winther-Jensen, M. Hilder, B. Winther-Jensen and D. R. MacFarlane, Catal. Today, 2013, 200, 36-40.

5 N. V. Plechkova and K. R. Seddon, Chem. Soc. Rev., 2008, 37, 123-150. 
6 E. G. Garcia, A. K. Ressmann, P. Gaertner, R. Zirbs, R. L. Mach, R. Krska, K. Bica and K. Brunner, Anal. Bioanal. Chem., 2014, 406, 7773-7784.

7 R. Zirbs, K. Strassl, P. Gaertner, C. Schroder and K. Bica, RSC Adv., 2013, 3, 26010-26016.

8 R. Hayes, G. G. Warr and R. Atkin, Chem. Rev., 2015, 115, 6357-6426.

9 A. Podgorsek, A. S. Pensado, C. C. Santini, M. F. C. Gomes and A. A. H. Padua, J. Phys. Chem. C, 2013, 117, 3537-3547.

10 P. S. Campbell, C. C. Santini, D. Bouchu, B. Fenet, K. Philippot, B. Chaudret, A. A. H. Padua and Y. Chauvin, Phys. Chem. Chem. Phys., 2010, 12, 4217-4223.

11 M. Anouti and J. Jacquemin, Colloids Surf., A, 2014, 445, 1-11.

12 P. Wang, S. M. Zakeeruddin, P. Comte, I. Exnar and M. Gratzel, J. Am. Chem. Soc., 2003, 125, 1166-1167.

13 Z. Zolek-Tryznowska, J. Izdebska and M. Golazbek, Color. Technol., 2014, 130, 314-318.

14 E. Vanecht, K. Binnemans, S. Patskovsky, M. Meunier, J. W. Seo, L. Stappers and J. Fransaer, Phys. Chem. Chem. Phys., 2012, 14, 5662-5671.

15 K. Richter, A. Birkner and A. V. Mudring, Phys. Chem. Chem. Phys., 2011, 13, 7136-7141.

16 L. L. Lazarus, C. T. Riche, N. Malmstadt and R. L. Brutchey, Langmuir, 2012, 28, 15987-15993.

17 G. Salas, A. Podgorsek, P. S. Campbell, C. C. Santini, A. A. H. Padua, M. F. C. Gomes, K. Philippot, B. Chaudret and M. Turmine, Phys. Chem. Chem. Phys., 2011, 13, 13527-13536.

18 C. Guibert, V. Dupuis, J. Fresnais and V. Peyre, J. Colloid Interface Sci., 2015, 454, 105-111.

19 J. A. Smith, O. Werzer, G. B. Webber, G. G. Warr and R. Atkin, J. Phys. Chem. Lett., 2010, 1, 64-68.

20 I. Szilagyi, T. Szabo, A. Desert, G. Trefalt, T. Oncsik and M. Borkovec, Phys. Chem. Chem. Phys., 2014, 16, 9515-9524.

21 K. Ueno, A. Inaba, M. Kondoh and M. Watanabe, Langmuir, 2008, 24, 5253-5259.

22 J. Nordstrom, L. Aguilera and A. Matic, Langmuir, 2012, 28, 4080-4085.

23 M. Mamusa, J. Siriex-Plenet, F. Cousin, E. Dubois and V. Peyrea, Soft Matter, 2014, 10, 1097-1101.

24 V. Valmacco, G. Trefalt, P. Maroni and M. Borkovec, Phys. Chem. Chem. Phys., 2015, 17, 16553-16559.

25 D. F. Evans and H. Wennerstrom, The Colloidal Domain, John Wiley, New York, 1999.

26 T. Oncsik, G. Trefalt, Z. Csendes, I. Szilagyi and M. Borkovec, Langmuir, 2014, 30, 733-741.

27 C. Schneider, M. Hanisch, B. Wedel, A. Jusufi and M. Ballauff, J. Colloid Interface Sci., 2011, 358, 62-67.

28 P. Lo Nostro and B. W. Ninham, Chem. Rev., 2012, 112, 2286-2322.

29 T. Oncsik, G. Trefalt, M. Borkovec and I. Szilagyi, Langmuir, 2015, 31, 3799-3807.

30 D. F. Parsons, M. Bostrom, P. Lo Nostro and B. W. Ninham, Phys. Chem. Chem. Phys., 2011, 13, 12352-12367.

31 T. Lopez-Leon, M. J. Santander-Ortega, J. L. Ortega-Vinuesa and D. Bastos-Gonzalez, J. Phys. Chem. C, 2008, 112, 16060-16069.
32 J. M. Peula-Garcia, J. L. Ortega-Vinuesa and D. BastosGonzalez, J. Phys. Chem. C, 2010, 114, 11133-11139.

33 N. Schwierz, D. Horinek and R. R. Netz, Langmuir, 2010, 26, 7370-7379.

34 C. Calero, J. Faraudo and D. Bastos-Gonzalez, J. Am. Chem. Soc., 2011, 133, 15025-15035.

35 M. Lund, R. Vacha and P. Jungwirth, Langmuir, 2008, 24, 3387-3391.

36 F. J. M. Ruiz-Cabello, G. Trefalt, T. Oncsik, I. Szilagyi, P. Maroni and M. Borkovec, J. Phys. Chem. B, 2015, 119, 8184-8193.

37 F. Dumont, J. Warlus and A. Watillon, J. Colloid Interface Sci., 1990, 138, 543-554.

38 R. Tian, G. Yang, H. Li, X. D. Gao, X. M. Liu, H. L. Zhu and Y. Tang, Phys. Chem. Chem. Phys., 2014, 16, 8828-8836.

39 C. Lange, G. Patil and R. Rudolph, Protein Sci., 2005, 14, 2693-2701.

40 Z. Yang, J. Biotechnol., 2009, 144, 12-22.

41 C. Sanfilippo, N. D’Antona and G. Nicolosi, Biotechnol. Lett., 2004, 26, 1815-1819.

42 D. Constantinescu, H. Weingartner and C. Herrmann, Angew. Chem., Int. Ed., 2007, 46, 8887-8889.

43 K. Fujita, D. R. MacFarlane and M. Forsyth, Chem. Commun., 2005, 4804-4806.

44 M. I. Mishchenko, L. D. Travis and A. A. Lacis, Scattering, Absorption, and Emission of Light by Small Particles, University Press, Cambridge, 2002.

45 H. Holthoff, S. U. Egelhaaf, M. Borkovec, P. Schurtenberger and H. Sticher, Langmuir, 1996, 12, 5541-5549.

46 K. L. Chen, S. E. Mylon and M. Elimelech, Langmuir, 2007, 23, 5920-5928.

47 A. Zaccone, H. Wu, M. Lattuada and M. Morbidelli, J. Phys. Chem. B, 2008, 112, 1976-1986.

48 M. Schudel, S. H. Behrens, H. Holthoff, R. Kretzschmar and M. Borkovec, J. Colloid Interface Sci., 1997, 196, 241-253.

49 S. H. Behrens, M. Borkovec and P. Schurtenberger, Langmuir, 1998, 14, 1951-1954.

50 M. Kobayashi, M. Skarba, P. Galletto, D. Cakara and M. Borkovec, J. Colloid Interface Sci., 2005, 292, 139-147.

51 M. Elimelech, J. Gregory, X. Jia and R. A. Williams, Particle Deposition and Aggregation: Measurement, Modeling, and Simulation, Butterworth-Heinemann Ltd., Oxford, 1995.

52 T. Lopez-Leon, J. L. Ortega-Vinuesa and D. Bastos-Gonzalez, ChemPhysChem, 2012, 13, 2382-2391.

53 A. Stoppa, J. Hunger, G. Hefter and R. Buchner, J. Phys. Chem. B, 2012, 116, 7509-7521.

54 R. Sadeghi and N. Ebrahimi, J. Phys. Chem. B, 2011, 115, 13227-13240.

55 M. Bester-Rogac, A. Stoppa, J. Hunger, G. Hefter and R. Buchner, Phys. Chem. Chem. Phys., 2011, 13, 17588-17598.

56 M. Bester-Rogac, J. Hunger, A. Stoppa and R. Buchner, J. Chem. Eng. Data, 2011, 56, 1261-1267.

57 H. K. Stassen, R. Ludwig, A. Wulf and J. Dupont, Chem. - Eur. J., 2015, 21, 8324-8335. 
58 M. Markiewicz, W. Mrozik, K. Rezwan, J. Thoming, J. Hupka and C. Jungnickel, Chemosphere, 2013, 90, 706-712.

59 U. Preiss, C. Jungnickel, J. Thoming, I. Krossing, J. Luczak, M. Diedenhofen and A. Klamt, Chem. - Eur. J., 2009, 15, 8880-8885.

60 J. Luczak, J. Hupka, J. Thoming and C. Jungnickel, Colloids Surf., A, 2008, 329, 125-133.

61 D. W. Fuerstenau and M. Colic, Colloids Surf., A, 1999, 146, 33-47.
62 A. Watanabe, Bull. Inst. Chem. Res., Kyoto Univ., 1960, 38, 179-215.

63 L. Liang and J. J. Morgan, Aquat. Sci., 1990, 52, 32-55.

64 R. H. Ottewill and M. C. Rastogi, Trans. Faraday Soc., 1960, 56, 866.

65 P. Sinha, I. Szilagyi, F. J. M. Ruiz-Cabello, P. Maroni and M. Borkovec, J. Phys. Chem. Lett., 2013, 4, 648-652.

66 I. Szilagyi, A. Polomska, D. Citherlet, A. Sadeghpour and M. Borkovec, J. Colloid Interface Sci., 2013, 392, 34-41. 